\title{
Asymmetric energetic costs in reciprocal-cross hybrids between carnivorous mice (Onychomys)
}

\author{
J. Ryan Shipley ${ }^{1}$, Polly Campbell ${ }^{2}$, Jeremy B. Searle ${ }^{1}$ and Bret Pasch ${ }^{1,3,4,5, *, \neq}$
}

\begin{abstract}
Aerobic respiration is a fundamental physiological trait dependent on coordinated interactions between gene products of the mitochondrial and nuclear genomes. Mitonuclear mismatch in interspecific hybrids may contribute to reproductive isolation by inducing reduced viability (or even complete inviability) due to increased metabolic costs. However, few studies have tested for effects of mitonuclear mismatch on respiration at the whole-organism level. We explored how hybridization affects metabolic rate in closely related species of grasshopper mice (genus Onychomys) to better understand the role of metabolic costs in reproductive isolation. We measured metabolic rate across a range of temperatures to calculate basal metabolic rate $(B M R)$ and cold-induced metabolic rate $\left(\mathrm{MR}_{\mathrm{c}}\right)$ in $\mathrm{O}$. leucogaster, $O$. torridus and $O$. arenicola, and in reciprocal $F_{1}$ hybrids between the latter two species. Within the genus, we found a negative correlation between mass-specific BMR and body mass. Although $O$. arenicola was smaller than $O$. torridus, hybrids from both directions of the cross resembled $O$. arenicola in body mass. In contrast, hybrid BMR was strongly influenced by the direction of the cross: reciprocal $F_{1}$ hybrids were different from each other but indistinguishable from the maternal species. In addition, $\mathrm{MR}_{\mathrm{c}}$ was not significantly different between hybrids and either parental species. These patterns indicate that metabolic costs are not increased in Onychomys $F_{1}$ hybrids and, while exposure of incompatibilities in $\mathrm{F}_{2}$ hybrids cannot be ruled out, suggest that mitonuclear mismatch does not act as a primary barrier to gene flow. Maternal matching of BMR is suggestive of a strong effect of mitochondrial genotype on metabolism in hybrids. Together, our findings provide insight into the metabolic consequences of hybridization, a topic that is understudied in mammals.
\end{abstract}

KEY WORDS: Mitonuclear mismatch, Metabolic rate, Interspecific hybrids, $\mathrm{F}_{1}$ hybrid, OXPHOS, Reproductive isolation

\section{INTRODUCTION}

Aerobic respiration depends on coordinated interaction between products of the mitochondrial and nuclear genomes. The interdependence of these two genomes is among the most ancient and taxonomically pervasive examples of coevolution (Lang et al., 1999; Rand et al., 2004). Energy in the form of ATP is generated via

\footnotetext{
${ }^{1}$ Department of Ecology and Evolutionary Biology, Cornell University, Ithaca, NY 14853, USA. ${ }^{2}$ Department of Integrative Biology, Oklahoma State University, Stillwater, OK 74078, USA. ${ }^{3}$ Bioacoustics Research Program, Cornell Lab of Ornithology, Cornell University, Ithaca, NY 14850, USA. ${ }^{4}$ Macaulay Library, Cornell Lab of Ornithology, Cornell University, Ithaca, NY 14850, USA. ${ }^{5}$ Department of Neurobiology and Behavior, Cornell University, Ithaca, NY 14853, USA.

*Present address: Department of Biological Sciences, Northern Arizona University, Flagstaff, AZ 86011, USA.

‡Author for correspondence (bret.pasch@nau.edu)
}

(D) B.P., 0000-0001-9168-6770 oxidative phosphorylation (OXPHOS) within the mitochondria (Alberts et al., 2014). Four of the five OXPHOS enzyme complexes comprise proteins of both nuclear and mitochondrial origin (Ryan and Hoogenraad, 2007; McKenzie et al., 2007), and mitochondrial DNA replication, repair and transcription all depend on nuclear genes (reviewed in Rand et al., 2004). While the identity of mitochondrial genes is highly conserved across Metazoa (Saccone et al., 2006), the high substitution rate of mitochondrial DNA can drive compensatory evolution in the nuclear genome on microevolutionary time scales (Wolff et al., 2014). For example, nuclear components of the cytochrome $c$ oxidase complex in primates (Osada and Akashi, 2012) and nuclear-encoded mitochondrial ribosome proteins in invertebrates exhibit accelerated evolution (Barreto and Burton, 2012). Because efficient mitochondrial function is fundamental to energy production, suboptimal mitochondrial-nuclear (mitonuclear) interactions are expected to have large negative effects on key physiological processes, including growth, development and fertility (Lane, 2011).

Interdependence, opportunity for rapid coevolution, and high fitness costs of inefficient respiration suggest that mismatches between mitochondrial and nuclear genomes have the potential to contribute to barriers to gene flow between closely related or incipient species (Gershoni et al., 2009; Johnson, 2010; Burton and Barreto, 2012; Wolff et al., 2014). This argument is consistent with the predictions of the Dobzhansky-Muller model for the evolution of postzygotic reproductive isolation, in which hybrid inviability and sterility are explained by faulty interactions between loci evolving on different genetic backgrounds (Bateson, 1909; Dobzhansky, 1937; Muller, 1942). Indeed, mitonuclear incompatibilities appear to contribute to speciation in copepods (Tigriopus californicus); in inter-population crosses, hybrid inviability is strongly associated with reduced efficiency of OXPHOS complexes and of nuclear-encoded mitochondrial transcription (Burton et al., 2006; Ellison and Burton, 2008, 2010). While such incompatibilities appear widespread in invertebrates (parasitoid wasps: Breeuwer and Werren, 1995; Niehuis et al., 2008; Ellison et al., 2008; nematodes: Chang et al., 2015; Drosophila: Meiklejohn et al., 2013) and yeast (Lee et al., 2008; Chou et al., 2010), further work is needed to assess the taxonomic scope and contribution of mitonuclear dysregulation to reproductive isolation.

In vertebrates, the effects of mitonuclear mismatch are surprisingly underexplored. The prevalence of $F_{1}$ hybrid inviability in sunfishes (Centrarchidae) is positively correlated with the rate of mitochondrial evolution in the maternal parental species (Bolnick et al., 2008). Similarly, analyses of mitochondrial function in mitonuclear hybrid cell lines in rodents (McKenzie et al., 2003) and primates (Kenyon and Moraes, 1997) indicate that mitochondrial OXPHOS efficiency decreases with increased genetic distance between the host nuclear genome and 
heterospecific mitochondria. At the organismal level, basal metabolic rate (BMR) differs among allopatric populations of the stonechat (Saxicola torquata), a Eurasian passerine, but is not dysregulated in interpopulation hybrids (Tieleman et al., 2009). In contrast, wild-caught chickadee hybrids (Poecile atricappillus $\times$ Poecile carolinensis; Olson et al., 2010) and labreared $\mathrm{F}_{1}$ crested newt hybrids (Triturus carnifex $\times$ Triturus dobrogicus; Gvoždík, 2012) exhibit significantly increased metabolic rates relative to parental species. Given that BMR represents the minimal cost of maintenance, such increased metabolic costs in hybrids translates into less energy available for other functions such as growth or reproduction (Lane, 2011). Furthermore, metabolic inefficiency should become more pronounced in animals under stress to meet energetic demands, such as maintaining homeothermy in low ambient temperatures. To our knowledge, the contribution of mitonuclear mismatch to the origin and maintenance of species boundaries is untested in mammals. Herein, using BMR and cold-induced metabolic rate $\left(\mathrm{MR}_{\mathrm{c}}\right)$ as indicators of mitochondrial efficiency, we tested for evidence of mitonuclear incompatibility in laboratory-reared grasshopper mice (genus Onychomys) and their interspecific $\mathrm{F}_{1}$ hybrids.

Grasshopper mice are carnivorous rodents that inhabit arid to semi-arid deserts, grasslands and prairies throughout western North America. Northern grasshopper mice (O. leucogaster) range throughout the Interior Plains and Columbia and Great Basins (26-49 g; McCarty, 1978), whereas Southern (O. torridus; 20$40 \mathrm{~g})$ and Chihuahuan grasshopper mice (O. arenicola; 20-35 g) primarily inhabit Sonoran and Chihuahuan desert, respectively (McCarty, 1975; Sullivan et al., 1986). In southwestern New Mexico, all three species come into contact. Here, O. leucogaster appears to segregate from its congeners based on a preference for more mesic environments (Findley et al., 1975). Although relatively cryptic based on external phenotype (Hinesley, 1979; Sullivan et al., 1986), $O$. arenicola and $O$. torridus are readily discriminated by karyotype, mitochondrial haplotypes and nuclear gene sequences (Riddle, 1995; Riddle and Honeycutt, 1990). In fact, molecular phylogenies place $O$. arenicola as sister to O. leucogaster (Riddle, 1995; Miller and Engstrom, 2008). Importantly, there is no evidence of historic mitochondrial introgression between any of the three species (P.C. and B.P., unpublished). $\mathrm{F}_{1}$ hybrids between $O$. arenicola and $O$. torridus occur in the contact zone but are extremely rare (Sullivan et al., 1986). Nonetheless, the genus can be interbred in the laboratory and there is anecdotal evidence for reduced viability in hybrids (Pinter, 1971). The system provides an excellent opportunity to examine whether abnormalities in hybrid energetics contribute to reproductive isolation.

We measured metabolic rates at a range of temperatures in all three Onychomys species to characterize the range of metabolic rates within the genus, and used reciprocal $\mathrm{F}_{1}$ hybrids between $O$. arenicola and $O$. torridus to explore the energetics of hybrid offspring and the genetic mechanisms mediating aerobic respiration. If mitonuclear compatibility regulates metabolism, we predicted that either hybrid BMR or hybrid $\mathrm{MR}_{\mathrm{c}}$ would be outside the range of both parental species ('mitochondrial-nuclear coadaptation' hypothesis; Blier et al., 2001; Rand et al., 2004; Gershoni et al., 2009; Fig. 1A). Given the low frequency of hybrids in nature and relatively high mitochondrial divergence between the two species (10\% for cytochrome $b$; Bradley et al., 2004; Miller and Engstrom, 2008), we hypothesized that mitonuclear incompatibility would reduce respiratory efficiency in hybrids, resulting in higher $\mathrm{BMR}$ or $\mathrm{MR}_{\mathrm{c}}$ compared with that of parental species (e.g. Olson et al., 2010; Lane, 2011; Gvoždík, 2012). In other words, hybridization would carry a metabolic cost. In contrast, if mitochondrial genes are the primary determinant of metabolism, we predicted that metabolic rate would differ between hybrids from reciprocal directions of the cross and would match BMR or $\mathrm{MR}_{\mathrm{c}}$ of the maternal species ('mitochondrial control' hypothesis; Tieleman et al., 2009; Fig. 1B). Finally, if metabolism is primarily under nuclear control, we predicted no difference in either metric between reciprocal $F_{1}$ hybrids and metabolic rates intermediate to those of both parent species ('nuclear control' hypothesis; Tieleman et al., 2009; Fig. 1C).

\section{MATERIALS AND METHODS \\ Animal housing}

Mice used in this study were the progeny of wild-caught O. leucogaster (Wied-Neuwied 1841), O. torridus (Coues 1874) and $O$. arenicola Mearns 1896 captured in the Animas Valley, New Mexico, USA, in June-August 2014 and transferred to animal facilities at Cornell University. Mice were housed in pairs for breeding, maintained on a $14 \mathrm{~h}: 10 \mathrm{~h}$ light:dark cycle $\left(21 \pm 2^{\circ} \mathrm{C}\right)$, and provided with rodent chow (LabDiet Prolab RMH 3000) and water ad libitum. Reciprocal $\mathrm{F}_{1}$ hybrids were produced by crossing $O$. torridus females with $O$. arenicola males (hereafter, $t$ Q $\times a \sigma^{\star}$ ) and $O$. arenicola females with $O$. torridus males (hereafter, $a$ Q $\times t{ }^{\star}$ ),
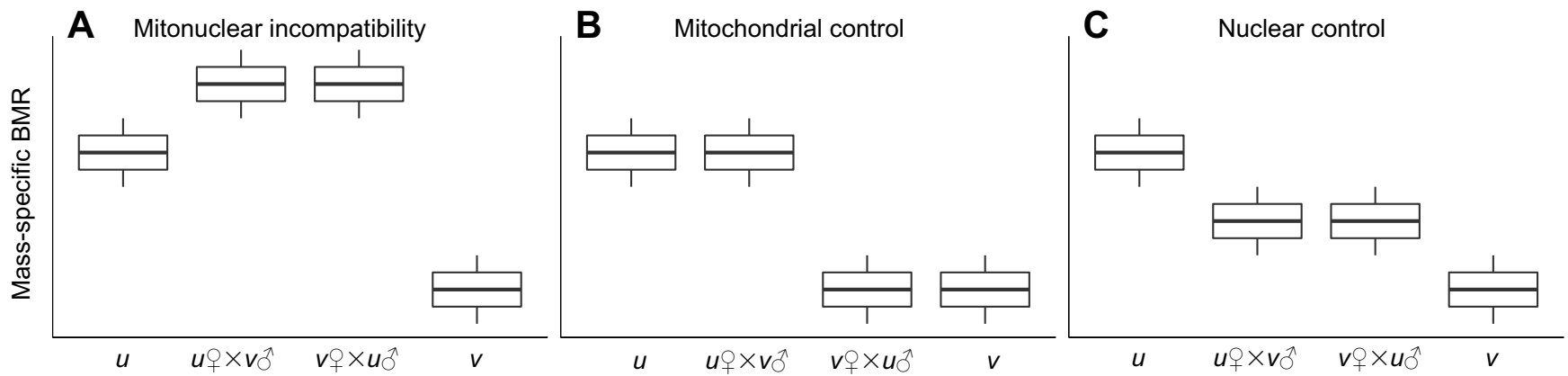

Fig. 1. Possible mechanisms that influence mitochondrial respiration in $F_{1}$ hybrids as illustrated with hypothetical results for parental species ( $u$ or $v$ )

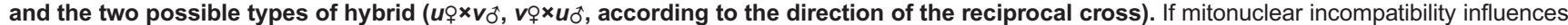
hybrid respiration (A), then basal metabolic rate (BMR) of hybrids should be greater than that of either parent species as a result of mismatch and reduced efficiency (Lane, 2011; Olson et al., 2010). However, if the mitochondrial genome controls respiration (B), then the hybrids should most closely resemble their maternal parent. If respiration is primarily under nuclear control $(C)$, then we expect no difference in BMR between reciprocal $F_{1}$ hybrids and metabolic rates intermediate to those of both parent species (Tieleman et al., 2009). 
resulting in offspring with equivalent hybrid nuclear genomes (except for the sex chromosomes in males) and mitochondrial DNA from either $O$. torridus or $O$. arenicola. Pups were weaned at 30 days and housed individually until respirometry experiments were performed. All individuals used in the respirometry experiments were adults ( $>5$ months) in non-reproductive condition. Animals were collected with approval from the New Mexico Department of Game and Fish (authorization no. 3562). The research was approved by IACUC (protocol no. 2014-0063) at Cornell University.

\section{Respirometry}

Respirometry trials were performed during the diurnal quiescent period (09:00 h-17:00 h) in December 2015. Access to food and water was restricted $3-5 \mathrm{~h}$ before initiation of respirometry to ensure that animals were post-absorptive (i.e. not actively digesting food). Individuals were acclimated to a starting ambient temperature $\left(T_{\mathrm{a}}\right)$ of $1-3.5^{\circ} \mathrm{C}$ for $2 \mathrm{~h}$ before measurements were collected. Measurements were taken at the initial temperature for $1 \mathrm{~h}$, then $T_{\mathrm{a}}$ was increased at $5^{\circ} \mathrm{C} \mathrm{h}^{-1}$, and maintained at a stable temperature for $1 \mathrm{~h}$ until a final $T_{\mathrm{a}}$ of $37-38^{\circ} \mathrm{C}$ was reached.

We measured resting metabolic rate (RMR) using a pull-mode flow-through respirometer coupled with a climate-controlled chamber (Lighton, 2008). Excurrent gas was analyzed using a Sable Systems FoxBox field $\mathrm{O}_{2} / \mathrm{CO}_{2}$ analyzer. Samples were scrubbed of water vapor before $\mathrm{CO}_{2}$ measurements using refurbished Drierite. Before the respirometry measurements, all Drierite was exposed to ambient air for a minimum of $2 \mathrm{~min}$, thereby reducing $\mathrm{CO}_{2}$ affinity and overall washout times (White et al., 2006). After $\mathrm{CO}_{2}$ measurements, the gas stream was scrubbed using a combination of scrubbers including Drierite-soda lime-Ascarite before measuring $\mathrm{O}_{2}$ concentration. Baseline measurements lasting $7 \mathrm{~min}$ were taken every $35 \mathrm{~min}$, and after the completion of the trial. Measured versus actual flow rates were corrected using the water displacement method (i.e. measuring the time for excurrent airflow to displace water in an inverted graduated cylinder of known volume; see Lighton and Halsey, 2011). A $3 \mathrm{~m}$ copper coil constructed of $6.35 \mathrm{~mm}$ i.d. tubing was placed in-line upstream inside the respiration chamber to equilibrate the temperature between the chamber and the incurrent airstream. All connection tubing was $6.35 \mathrm{~mm}$ i.d. Bev-a-line IV.

Raw $\mathrm{O}_{2}$ and $\mathrm{CO}_{2}$ measurements were first drift-corrected (3rd degree polynomial - cubic Hermite spline) using baseline data.
Using the standard temperature and pressure (STP)-compensated flow rate, oxygen consumption $\left(\dot{V}_{\mathrm{O}_{2}}\right)$ was calculated as:

$$
\dot{V}_{\mathrm{O}_{2}}=\mathrm{FR} \times\left(F_{\mathrm{I}_{\mathrm{O}_{2}}}-F_{\mathrm{E}_{\mathrm{O}_{2}}}\right) /\left(1-F_{\mathrm{I}_{\mathrm{O}_{2}}}\right)
$$

and carbon dioxide production as:

$$
\dot{V}_{\mathrm{CO}_{2}}=\left[\mathrm{FR}\left(F \mathrm{E}_{\mathrm{CO}_{2}}-F_{\mathrm{I}_{\mathrm{CO}_{2}}}\right)-F_{\mathrm{E}_{\mathrm{CO}_{2}}}\left(\dot{V}_{\mathrm{O}_{2}}\right)\right] /\left(1-F \mathrm{E}_{\mathrm{CO}_{2}}\right),
$$

where $\mathrm{FR}$ is the corrected incurrent flow rate, $F_{\mathrm{I}_{2}} / F_{\mathrm{I}_{\mathrm{CO}}}$ is the fractional incurrent gas concentration and $\mathrm{FE}_{\mathrm{O}_{2}} / \mathrm{F}_{\mathrm{E}_{\mathrm{CO}_{2}}}$ is the fractional excurrent gas concentration.

\section{Statistical analyses}

BMR was calculated as the lowest stable value for $\dot{V}_{\mathrm{O}_{2}}$ between 30 and $32^{\circ} \mathrm{C}$, within the previously published limits of the thermoneutral zone in Onychomys (Whitford and Conley, 1971). $\mathrm{MR}_{\mathrm{c}}$ was calculated as the predicted RMR for each individual at $0^{\circ} \mathrm{C}$. We used the RMR measurements below BMR to derive a linear equation for each individual. The starting temperature for each respirometry trial was between 1 and $3.5^{\circ} \mathrm{C}$; thus, we were able to interpolate metabolic rates for all individuals at $0^{\circ} \mathrm{C}$. Finally, we re-ran respirometry trials on an individual from each of the three species and one $\mathrm{F}_{1}$ hybrid $\left(t\right.$ o $\left.\times a \sigma^{*}\right)$ a minimum of 5 days after the initial trial to test for repeatability of our metabolic measurements. The effect of species $(O$. leucogaster, $O$. torridus, $O$. arenicola $)$ or reciprocal $F_{1}$ hybrid identity on body mass and on mass-specific BMR was tested with one-way ANOVA. All pairwise comparisons were evaluated with post hoc Tukey's HSD tests. Analyses were performed using R 3.2.2 (R Development Core Team 2015).

\section{RESULTS}

\section{Body mass}

Overall, body mass differed among $O$. arenicola, $O$. leucogaster, $O$. torridus and reciprocal $\mathrm{F}_{1}$ hybrids between $O$. arenicola and $O$. torridus (ANOVA, $F_{4,15}=16.48, P<0.001$; Fig. 2A). Both $F_{1}$ hybrid crosses were smaller than $O$. leucogaster and $O$. torridus (Tukey HSD, $P<0.01$, all comparisons), yet were indistinguishable from each other and from $O$. arenicola (Tukey HSD, $P=0.73,0.78$ and 1.0, respectively; Table 1).

\section{Metabolic rates}

BMR varied with body mass, explained by an exponential curve with the following relationship:

$$
\mathrm{BMR}=-0.0166 \times M+1.6829,
$$
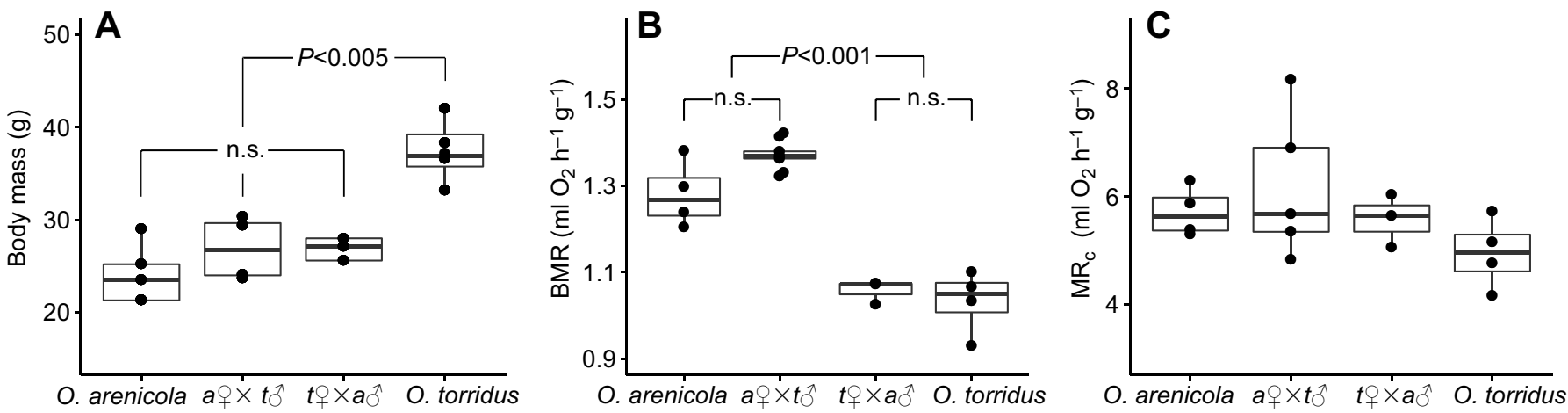

Fig. 2. Size and energetics comparison of parental and hybrid Onychomys. (A) Body mass, (B) mass-specific BMR and (C) mass-specific cold-induced metabolic rate $\left(\mathrm{MR}_{\mathrm{c}}\right)$ of Onychomys arenicola, Onychomys torridus and reciprocal hybrids. The mass of $\mathrm{O}$. torridus is significantly different from that of the other parent species, $O$. arenicola, and the reciprocal hybrid crosses (GLM, Tukey HSD, $P<0.005)$. The BMR of each hybrid most closely resembles the BMR of the female parent species. Sample size: $O$. arenicola, $N=4$; O. torridus, $N=4$; a 
Table 1. Body mass, whole-organism and mass-specific basal metabolic rate (BMR), and mass-specific cold-induced metabolic rate (MR $\mathrm{R}_{\mathrm{c}}$ ) for 3 species and 2 hybrid crosses of Onychomys

\begin{tabular}{|c|c|c|c|c|c|c|}
\hline Species or hybrid cross & $N$ & $n$ & Body mass (g) & $\begin{array}{l}\text { Whole-organism } \\
\text { BMR }\left(\mathrm{ml} \mathrm{O}_{2} \mathrm{~h}^{-1}\right)\end{array}$ & $\begin{array}{l}\text { Mass-specific } \\
\text { BMR }\left(\mathrm{ml} \mathrm{O}_{2} \mathrm{~h}^{-1} \mathrm{~g}^{-1}\right)\end{array}$ & $\begin{array}{l}\text { Mass-specific } \\
\operatorname{MR}_{\mathrm{c}}\left(\mathrm{ml} \mathrm{O}_{2} \mathrm{~h}^{-1} \mathrm{~g}^{-1}\right)\end{array}$ \\
\hline O. arenicola (a) & 4 & 5 & $26.90 \pm 3.49$ & $33.30 \pm 3.05$ & $1.28 \pm 0.08$ & $5.72 \pm 0.45$ \\
\hline$a o \times t \sigma$ & 5 & 5 & $26.92 \pm 1.21$ & $28.43 \pm 0.81$ & $1.37 \pm 0.03$ & $6.18 \pm 1.34$ \\
\hline$t+x_{a} 0^{-}$ & 3 & 4 & $24.06 \pm 3.21$ & $32.91 \pm 3.95$ & $1.06 \pm 0.03$ & $5.57 \pm 0.49$ \\
\hline O. torridus $(t)$ & 4 & 5 & $37.25 \pm 3.62$ & $37.45 \pm 2.32$ & $1.03 \pm 0.07$ & $4.95 \pm 0.65$ \\
\hline O. leucogaster & 4 & 5 & $38.87 \pm 4.30$ & $40.94 \pm 4.88$ & $1.05 \pm 0.03$ & $4.17 \pm 0.56$ \\
\hline
\end{tabular}

$N$, number of individuals; $n$, number of respirometry trials. Values are means \pm s.d.

where $M$ is body mass $\left(r^{2}=0.78\right)$. There was a significant overall effect of species/hybrid identity on whole-organism BMR and mass-specific BMR (ANOVA, $F_{4,15}=6.851, \quad P<0.01$ and $F_{4,15}=30.36, \quad P<0.001$, respectively; Fig. 2B). Onychomys arenicola had a higher mass-specific BMR than that of either $O$. torridus or $O$. leucogaster (Tukey HSD, both $P<0.005$ ), whereas there was no difference in mass-specific BMR between $O$. leucogaster and $O$. torridus (Tukey HSD, $P=0.98$; Table 1). Mass-specific BMR was significantly different between the two hybrid crosses (Tukey HSD, $P<0.001$ ) and from their paternal parental species (Tukey HSD: $t$ 우 $\times a$ ô versus $O$. arenicola, $P<0.001$; $a$ $\times t_{0} \overrightarrow{0}$ versus $O$. torridus, $\left.P<0.001\right)$, but was indistinguishable from their maternal parental species $\left(t\right.$ 우 $\times \mathrm{a}^{\dagger}$ versus $O$. torridus, $P=0.97$;

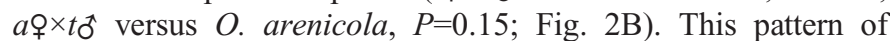
maternal matching in hybrids is consistent with the predictions of the mitochondrial control hypothesis (Fig. 1B).

We also found a significant overall effect of species/hybrid identity on mass-specific $\mathrm{MR}_{\mathrm{c}}\left(F_{4,15}=3.715, P=0.03\right)$ but not on whole-organism $\mathrm{MR}_{\mathrm{c}}$ (ANOVA, $F_{4,15}=1.474, P=0.26$; Fig. 2C). Mass-specific $\mathrm{MR}_{\mathrm{c}}$ was greater in $a$ Q $\times t^{-}$hybrids than in $O$. leucogaster (Tukey HSD, $P=0.02$; Table 1 ), but was not different for any other pairwise comparison (all $P>0.2$; Fig. $2 \mathrm{C}$ ).

Metabolic rate measurements showed strong repeatability for all individuals when compared with the original measurements ( $F_{1,6}=714.89, P<0.001, R=0.90$; Lessells and Boag, 1987).

\section{DISCUSSION}

We tested for evidence of mitonuclear incompatibility in the laboratoryreared $F_{1}$ hybrid progeny of two species of grasshopper mice from a contact zone where hybrids occur at very low frequencies. Mice from reciprocal hybrid crosses differed from each other in mass-specific BMR but not in body mass or $\mathrm{MR}_{\mathrm{c}}$. Hybrid values for these phenotypes were within the range of variation found within parental species and the genus as a whole. Thus, we did not find consistent evidence of respiratory inefficiency with either whole-organism or mass-specific metabolic rates. This suggests that mitonuclear incompatibilities are not expressed in Onychomys $\mathrm{F}_{1}$ hybrids. In addition, our results suggest that hybrid metabolic rate is maternally inherited, with no detectable influence of the $F_{1}$ nuclear genome. These findings provide evidence that the mitochondrial genome is a primary regulator of energy metabolism in Onychomys. In contrast, body mass of both hybrids was indistinguishable from that of the smaller parental species, $O$. arenicola. This result was also unexpected, as it is suggestive of a dominant inheritance pattern for a trait that prior studies in wild rodents have found to be determined by either additive inheritance (e.g. Sadowska et al., 2005) or maternal effects (e.g. Nespolo et al., 2003, 2005). We discuss the BMR and body mass results in turn.

\section{No evidence for metabolic costs in $F_{1}$ hybrids}

The potential for rapid coevolution between mitochondrial and nuclear genomes and the essential role of mitochondria in energy production is expected to yield reduced fitness when the two genomes are mismatched in hybrids (Gershoni et al., 2009; Johnson, 2010; Burton and Barreto, 2012). If nuclear genes that contribute to mitonuclear dysregulation are dominant-acting in hybrids, then incompatibilities should be exposed in $\mathrm{F}_{1}$ animals. Indeed, this seems to be the case in crested newt hybrids (Triturus species), in which $\mathrm{BMR}$ of $\mathrm{F}_{1}$ hybrids is significantly elevated relative to that of both parent species, consistent with reduced metabolic efficiency due to mitonuclear mismatch (Gvoždík, 2012). In contrast, $\mathrm{F}_{1}$ Onychomys hybrids appear to suffer no metabolic deficits, with individuals exhibiting equal or lower metabolic rates than expected based on body mass. This result suggests that mitonuclear incompatibility does not explain the rarity of natural hybrids between $O$. torridus and $O$. arenicola. There are, however, two important caveats to this conclusion.

First, we cannot rule out effects on hybrid metabolism beyond the $\mathrm{F}_{1}$ generation. Notably, the best-documented cases of mitonuclear incompatibility involve the exposure of recessive-acting incompatibilities in the nuclear genome. In both copepods (T. californicus) and parasitoid wasps (Nasonia species), $\mathrm{F}_{1}$ hybrids are normal and inviability associated with mitonuclear dysfunction is not expressed until the $\mathrm{F}_{2}$ generation (Burton et al., 2006; Niehuis et al., 2008; Ellison et al., 2008; Ellison and Burton, 2008). Future experiments using hybrids with either $O$. torridus or $O$. arenicola mitochondrial DNA on an $\mathrm{F}_{2}$ background will be required to determine whether mitonuclear-associated hybrid breakdown occurs in Onychomys.

Second, despite the lack of evidence for metabolic inefficiency when the organism is subjected to low temperatures, it is possible that exercise-induced stress (e.g. summit metabolism, $\dot{V}_{\mathrm{O}_{2}, \max }$ )

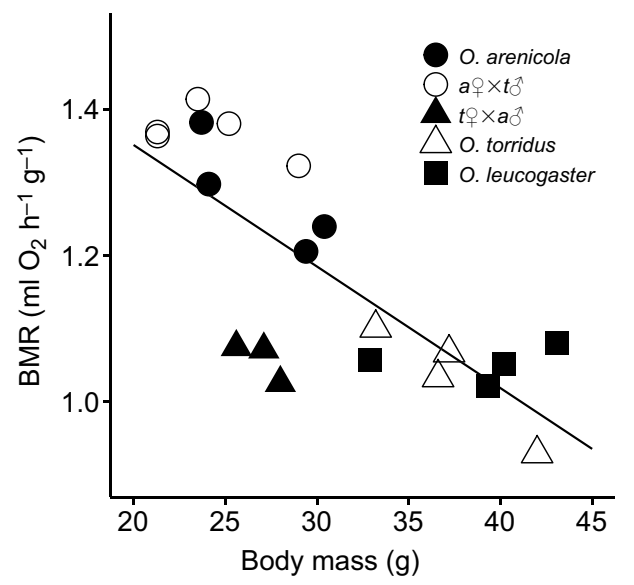

Fig. 3. Mass-specific BMR versus body mass in the genus Onychomys. The reciprocal $F_{1}$ hybrids each resemble their female parent in mass-specific BMR but the smaller parental species, $O$. arenicola, in body mass. Onychomys leucogaster is included for comparison. Sample size: $O$. arenicola, $N=4$; O. leucogaster, $N=4$; O. torridus, $N=4$; aำtô, $N=5$; to $\times a{ }^{\star}, N=3$. 
would uncover respiratory defects in $\mathrm{F}_{1}$ hybrids. For example, while gross metabolic function is normal in Mus musculus domesticusderived lab mice with mitochondria from a closely related congener, Mus spretus (McKenzie et al., 2004), these mitochondrial hybrids (cybrids) tire faster than controls in a running to exhaustion test (Nagao et al., 1998). A 2-fold excess in lactate production indicative of inefficient cellular metabolism may explain cybrid physical performance deficits (McKenzie et al., 2004). Given that mitochondrial divergence between $O$. torridus and $O$. arenicola is higher than that between M. m. domesticus and M. spretus (Suzuki et al., 2004), testing physiological performance limits in Onychomys hybrids would be of considerable interest.

\section{Evidence for mitochondrial effects on BMR}

Understanding the genetic architecture of bioenergetic traits is a central focus in evolutionary physiology (Hayes and Garland, 1995; Storz et al., 2015). The pattern of maternally matched BMR in reciprocal $\mathrm{F}_{1}$ hybrids reported here is consistent with mitochondrial control of energy metabolism in grasshopper mice (Fig. 1B; Tieleman et al., 2009). Given the importance of a large number of nuclear genes to mitochondrial function, this is a surprising result. In the absence of evidence for metabolic costs in hybrids, we would expect hybrid BMR to fall between that of the two parent species (Fig. 1C), as seen in most inter-population crosses in stonechats (Tieleman et al., 2009). Indeed, we are aware of only one other report of a similar signal of mitochondrial determination of BMR: analysis of a suite of metabolic parameters, including BMR, in the offspring of laboratory and wild house mice (M. m. domesticus) showed a significant effect of cross direction on BMR and other metabolic parameters (Richardson et al., 1994).

An alternative interpretation is that asymmetric BMR in Onychomys hybrids is due to maternal effects (the impact of maternal genotype or phenotype on offspring phenotype, Wolf and Wade, 2009; see also Lynch and Walsh, 1998). While the current experiment cannot discriminate between mitochondrial and maternal effects, quantitative genetic analyses of metabolic traits in other endotherms have not detected effects of maternal environment on BMR (Nespolo et al., 2003, 2005; Sadowska et al., 2005; Tieleman et al., 2009). Finally, phenotypes that depend on the direction of a cross are consistent with parent-of-origin effects, characteristic of imprinted genes. Although we are not aware of any direct connection between imprinted genes and BMR, several imprinted genes are highly expressed in the hypothalamus, a brain region critical to thermoregulation and energy homeostasis (Wilkinson et al., 2007). Ultimately, separating the proposed contribution of mitochondrial genotype to hybrid metabolism from that of maternal or parent-of-origin effects will require production of reciprocal cybrid lines: mice with $O$. arenicola mitochondria on an $O$. torridus background and vice versa.

\section{Effects of hybridization on body mass}

In contrast to hybrid mass-specific BMR, hybrid body mass was independent of the direction of the reciprocal cross: all hybrids resembled $O$. arenicola, the smaller of the two parent species. This result was surprising for two reasons. First, additive effects of many genes typically explain the heritable component of adult body mass in rodents (Cheverud et al., 1996; Gray et al., 2015), an inheritance pattern that should produce intermediate phenotypes in the offspring of a cross between different-sized parents. Non-additive effects on body mass, when present, are either maternal (e.g. Nespolo et al., 2005) or parent-of-origin dependent (e.g. Wolf et al., 2008). In both cases, offspring body mass depends on the direction of the reciprocal cross. Body mass in hybrid grasshopper mice did not match either of these genetic scenarios; the pattern we observed was consistent with dominant effects of $O$. arenicola nuclear genes. Second, regardless of the underlying genetic mechanism, the combination of invariant body mass and mitochondrially matched BMR resulted in hybrids with mass-specific BMR differing by greater than $40 \%$ in the reciprocal crosses. Specifically, BMR in hybrids with $O$. torridus mitochondria was low relative to body mass (Fig. 3). From a strictly energetic perspective, this metabolic phenotype could be advantageous under food-limited conditions but might negatively impact fitness when maximal energy expenditure is required (e.g. gestation and lactation; Derting, 1989; Koteja, 2000; Burton et al., 2011; Sadowska et al., 2013). Determining whether these trade-offs exist in $t$ 우 $\times a \sigma^{t}$ hybrids will require manipulation of food intake in reproductive and nonreproductive animals (e.g. Derting, 1989).

\section{Conclusions}

We found that mitochondrial and metabolic divergence between two species of grasshopper mice does not result in inefficient basal metabolism in $\mathrm{F}_{1}$ hybrids. Thus, while mitonuclear mismatch may affect hybrid phenotypes or generations (e.g. $F_{2}$ ) not studied here, our current results suggest that mitonuclear incompatibility does not explain the rarity of Onychomys hybrids in nature. Instead, our results can be interpreted as a strong effect of mitochondrial, but not nuclear, genotype on hybrid metabolic phenotype. Given the rarity of tests for metabolic costs of hybridization in endotherms, this study represents an important first step towards understanding the importance of mitonuclear incompatibilities to the evolution of reproductive isolation in mammals relative to other taxonomic groups. Future studies that focus on mitonuclear interactions in natural hybrid systems will help reveal the prevalence of incompatibilities in contributing to reproductive isolation in wild populations.

\section{Acknowledgements}

We thank M. Wilson and D. Zhao for assistance in animal husbandry, and J. E. Wallace and A. Cronin for help in capturing animals in the field.

\section{Competing interests}

The authors declare no competing or financial interests.

\section{Author contributions}

J.R.S. and B.P. conceived the study; J.B.S. and B.P. organized permits/logistics; J.R.S. and B.P. designed and performed the experiments; J.R.S., P.C. and B.P. analyzed the data; J.R.S., P.C., J.B.S. and B.P. wrote the manuscript.

\section{Funding}

The research was supported by a postdoctoral fellowship from the Cornell Lab of Ornithology to B.P.

\section{Data availability}

Data are available from the Dryad Digital Repository: http://dx.doi.org/10.5061/ dryad.22k3c (Shipley et al., 2016).

\section{References}

Alberts, B., Johnson, A., Lewis, J., Raff, M., Roberts, K. and Walter, P. (2014) Molecular Biology of the Cell, 6th edn. New York: Garland Science.

Barreto, F. S. and Burton, R. S. (2012). Evidence for compensatory evolution of ribosomal proteins in response to rapid divergence of mitochondrial rRNA. Mol. Biol. Evol. 30, 310-314.

Bateson, W. (1909). Heredity and variation in modern lights. In Darwin and Modern Science (ed. A. C. Seward), pp. 85-101. Cambridge, UK: Cambridge University Press. Blier, P. U., Dufresne, F. and Burton, R. S. (2001). Natural selection and the evolution of mtDNA-encoded peptides: evidence for intergenomic co-adaptation. Trends Genet. 17, 400-406.

Bolnick, D. I., Turelli, M., López-Fernández, H., Wainwright, P. C. and Near, T. J. (2008). Accelerated mitochondrial evolution and "Darwin's corollary": asymmetric viability of reciprocal $F_{1}$ hybrids in centrarchid fishes. Genetics 178, 1037-1048. 
Bradley, R. D., Edwards, C. W., Carroll, D. S. and Kilpatrick, C. W. (2004) Phylogenetic relationships of neotomine-peromyscine rodents: based on DNA sequences from the mitochondrial cytochrome-b gene. J. Mamm. 85, 389-395

Breeuwer, J. A. J. and Werren, J. H. (1995). Hybrid breakdown between two haplodiploid species: the role of nuclear and cytoplasmic genes. Evolution 49 705-717.

Burton, R. S. and Barreto, F. S. (2012). A disproportionate role for mtDNA in Dobzhansky-Muller incompatibilities? Mol. Ecol. 21, 4942-4957.

Burton, R. S., Ellison, C. K. and Harrison, J. S. (2006). The sorry state of F2 hybrids: consequences of rapid mitochondrial DNA evolution in allopatric populations. Am. Nat. 168, S14-S24.

Burton, T., Killen, S. S., Armstrong, J. D. and Metcalfe, N. B. (2011). What causes intraspecific variation in resting metabolic rate and what are its ecological consequences? Proc. R. Soc. B Biol. Sci. 278, 3465-3473.

Chang, C.-C., Rodriguez, J. and Ross, J. (2015). Mitochondrial-nuclear epistasis impacts fitness and mitochondrial physiology of interpopulation Caenorhabditis briggsae hybrids. G3 6, 209-219.

Cheverud, J. M., Routman, E. J., Duarte, F. A. M., van Swinderen, B., Cothran, K and Perel, C. (1996). Quantitative trait loci for murine growth. Genetics 142 1305-1319.

Chou, J.-Y., Hung, Y.-S., Lin, K.-H., Lee, H.-Y. and Leu, J.-Y. (2010). Multiple molecular mechanisms cause reproductive isolation between three yeast species. PLoS Biol. 8, e1000432.

Derting, T. L. (1989). Metabolism and food availability as regulators of production in juvenile cotton rats. Ecology 70, 587-595.

Dobzhansky, T. (1937). Genetics and the Origin of Species. New York: Columbia University Press.

Ellison, C. K. and Burton, R. S. (2008). Interpopulation hybrid breakdown maps to the mitochondrial genome. Evolution 62, 631-638.

Ellison, C. K. and Burton, R. S. (2010). Cytonuclear conflict in interpopulation hybrids: the role of RNA polymerase in mtDNA transcription and replication. J. Evol. Biol. 23, 528-538.

Ellison, C. K., Niehuis, O. and Gadau, J. (2008). Hybrid breakdown and mitochondrial dysfunction in hybrids of Nasonia parasitoid wasps. J. Evol. Biol. 21, 1844-1851.

Findley, J. S., Harris, A. H., Wilson, D. E. and Jones, C. (1975). Mammals of New Mexico. Albuquerque, NM: University of New Mexico Press.

Gershoni, M., Templeton, A. R. and Mishmar, D. (2009). Mitochondrial bioenergetics as a major motive force of speciation. BioEssays 31, 642-650.

Gray, M. M., Parmenter, M. D., Hogan, C. A., Ford, I., Cuthbert, R. J., Ryan, P. G. Broman, K. W. and Payseur, B. A. (2015). Genetics of rapid and extreme size evolution in island mice. Genetics 201, 213-228.

Gvoždík, L. (2012). Metabolic costs of hybridization in newts. Folia Zool. 61, 197.

Hayes, J. P. and Garland, T. (1995). The evolution of endothermy: testing the aerobic capacity model. Evolution 49, 836-847.

Hinesley, L. L. (1979). Systematics and distribution of two chromosome forms in the southern grasshopper mouse, genus Onychomys. J. Mamm. 60, 117-128.

Johnson, N. A. (2010). Hybrid incompatibility genes: remnants of a genomic battlefield? Trend. Genet. 26, 317-325.

Kenyon, L. and Moraes, C. T. (1997). Expanding the functional human mitochondrial DNA database by the establishment of primate xenomitochondrial cybrids. Proc. Natl. Acad. Sci. USA 94, 9131-9135.

Koteja, P. (2000). Energy assimilation, parental care and the evolution of endothermy. Proc. R. Soc. B Biol. Sci. 267, 479-484.

Lane, N. (2011). Mitonuclear match: optimizing fitness and fertility over generations drives ageing within generations. Bioessays 33, 860-869.

Lang, B. F., Gray, M. W. and Burger, G. (1999). Mitochondrial genome evolution and the origin of eukaryotes. Annu. Rev. Genet. 33, 351-397.

Lee, H.-Y., Chou, J.-Y., Cheong, L., Chang, N.-H., Yang, S.-Y. and Leu, J.-Y. (2008). Incompatibility of nuclear and mitochondrial genomes causes hybrid sterility between two yeast species. Cell 135, 1065-1073.

Lessells, C. M. and Boag, P. T. (1987). Unrepeatable repeatabilities: a common mistake. Auk 104, 116-121.

Lighton, J. R. B. (2008). Measuring Metabolic Rates: A Manual for Scientists Oxford, UK: Oxford University Press.

Lighton, J. R. B. and Halsey, L. G. (2011). Flow-through respirometry applied to Chamber systems: pros and cons, hints and tips. Comp. Biochem. Physiol. A Mol. Integr. Physiol. 158, 265-275.

Lynch, M. and Walsh, B. (1998). Genetic Analysis of Quantitative Traits. Sunderland, MA: Sinauer Associates.

McCarty, R. (1975). Onychomys torridus. Mamm. Spp. 59, 1-5.

McCarty, R. (1978). Onychomys leucogaster. Mamm. Spp. 87, 1-6.

McKenzie, M., Chiotis, M., Pinkert, C. A. and Trounce, I. A. (2003). Functional respiratory chain analyses in murid xenomitochondrial cybrids expose coevolutionary constraints of cytochrome $b$ and nuclear subunits of complex III. Mol. Biol. Evol. 20, 1117-1124.

McKenzie, M., Trounce, I. A., Cassar, C. A. and Pinkert, C. A. (2004). Production of homoplasmic xenomitochondrial mice. Proc. Natl Acad. Sci. USA 101 $1685-1690$
McKenzie, M., Lazarou, M., Thorburn, D. R. and Ryan, M. T. (2007). Analysis of mitochondrial subunit assembly into respiratory chain complexes using Blue Native polyacrylamide gel electrophoresis. Anal. Biochem. 364, 128-137.

Meiklejohn, C. D., Holmbeck, M. A., Siddiq, M. A., Abt, D. N., Rand, D. M. and Montooth, K. L. (2013). An incompatibility between a mitochondrial tRNA and its nuclear-encoded tRNA synthetase compromises development and fitness in Drosophila. PLoS Genet. 9, e1003238.

Miller, J. R. and Engstrom, M. D. (2008). The relationships of major lineages within peromyscine rodents: a molecular phylogenetic hypothesis and systematic reappraisal. J. Mamm. 89, 1279-1295.

Muller, H. J. (1942). Isolating mechanisms, evolution and temperature. Biol. Sympos. 6, 71-125.

Nagao, Y., Totsuka, Y., Atomi, Y., Kaneda, H., Lindahl, K. F., Imai, H. and Yonekawa, H. (1998). Decreased physical performance of congenic mice with mismatch between the nuclear and the mitochondrial genome. Genes Genet. Sys. 73, 21-27

Nespolo, R. F., Bacigalupe, L. D. and Bozinovic, F. (2003). Heritability of energetics in a wild mammal, the leaf-eared mouse (Phyllotis darwini). Evolution 57, 1679-1688.

Nespolo, R. F., Bustamante, D. M., Bacigalupe, L. D. and Bozinovic, F. (2005) Quantitative genetics of bioenergetics and growth-related traits in the wild mammal, Phyllotis darwini. Evolution 59, 1829-1837.

Niehuis, O., Judson, A. K. and Gadau, J. (2008). Cytonuclear genic incompatibilities cause increased mortality in male F2 hybrids of Nasonia giraulti and Nasonia vitripennis. Genetics 178, 413-426.

Olson, J. R., Cooper, S. J., Swanson, D. L., Braun, M. J. and Williams, J. B. (2010). The relationship of metabolic performance and distribution in Blackcapped and Carolina chickadees. Physiol. Biochem. Zool. 83, 263-275.

Osada, N. and Akashi, H. (2012). Mitochondrial-nuclear interactions and accelerated compensatory evolution: evidence from the primate cytochrome $c$ oxidase complex. Mol. Biol. Evol. 29, 337-346.

Pinter, A. J. (1971). Hybridization between two species of grasshopper mice (Onychomys) in the laboratory. J. Mamm. 52, 573-582.

Rand, D. M., Haney, R. A. and Fry, A. J. (2004). Cytonuclear coevolution: the genomics of cooperation. Trends Ecol. Evol. 19, 645-653.

Richardson, C. S., Dohm, M. R. and Garland, T. (1994). Metabolism and thermoregulation in crosses between wild and random-bred laboratory house mice (Mus domesticus). Physiol. Zool. 67, 944-975

Riddle, B. R. (1995). Molecular biogeography in the pocket mice (Perognathus and Chaetodipus) and grasshopper mice (Onychomys): the late Cenozoic development of a North American aridlands rodent guild. J. Mamm. 76, 283-301.

Riddle, B. R. and Honeycutt, R. L. (1990). Historical biogeography in the North American arid regions: an approach using mitochondrial-DNA phylogeny in grasshopper mice (genus Onychomys). Evolution 44, 1-15

Ryan, M. T. and Hoogenraad, N. J. (2007). Mitochondrial-nuclear communications Ann. Rev. Biochem. 76, 701-722.

Saccone, C., Lanave, C. and De Grassi, A. (2006). Metazoan OXPHOS gene families: evolutionary forces at the level of mitochondrial and nuclear genomes. Biochim. Biophys. Acta Bioenerget. 1757, 1171-1178.

Sadowska, E. T., Labocha, M. K., Baliga, K., Stanisz, A., Wróblewska, A. K. Jagusiak, W. and Kotega, P. (2005). Genetic correlations between basal and maximum metabolic rates in a wild rodent: consequences for evolution of endothermy. Evolution 59, 672-681.

Sadowska, J., Gẹbczyński, A. K. and Konarzewski, M. (2013). Basal metabolic rate is positively correlated with parental investment in laboratory mice. Proc. R. Soc. B Biol. Sci. 280, 20122576.

Shipley, J. R., Campbell, P., Searle, J. B. and Pasch, B. (2016). Data from: Asymmetric energetic costs in reciprocal-cross hybrids between carnivorous mice (Onychomys). Dryad Digital Repository. http://dx.doi.org.10.5061/dryad.22k3c.

Storz, J. F., Bridgham, J. T., Kelly, S. A. and Garland, T. (2015). Genetic approaches in comparative and evolutionary physiology. Am. J. Physiol. Regul. Integr. Comp. Physiol. 309, R197-R214.

Sullivan, R. M., Hafner, D. J. and Yates, T. L. (1986). Genetics of a contact zone between three chromosomal forms of the grasshopper mouse (genus Onychomys): a reassessment. J. Mamm. 67, 640-659.

Suzuki, H. Shimada, T., Terashima, M., Tsuchiya, K and Aplin, K. (2004) Temporal, spatial, and ecological modes of evolution of Eurasian Mus based on mitochondrial and nuclear gene sequences. Mol. Phylogenet. Evol. 33, 626-646.

Tieleman, B. I., Versteegh, M. A., Fries, A., Helm, B., Dingemanse, N. J., Gibbs, H. L. and Williams, J. B. (2009). Genetic modulation of energy metabolism in birds through mitochondrial function. Proc. R. Soc. B Biol. Sci. 276, 1685-1693.

White, C. R., Portugal, S. J., Martin, G. R. and Butler, P. J. (2006). Respirometry: anhydrous drierite equilibrates with carbon dioxide and increases washout times. Physiol. Biochem. Zool. 79, 977-980.

Whitford, W. G. and Conley, M. I. (1971). Oxygen consumption and wate metabolism in a carnivorous mouse. Comp. Biochem. Physiol. 40A, 797-803.

Wilkinson, L. S., Davies, W. and Isles, A. R. (2007). Genomic imprinting effects on brain development and function. Nat. Rev. Neurosci. 8, 832-843.

Wolf, J. B. and Wade, M. J. (2009). What are maternal effects (and what are they not)? Proc. R. Soc. B Biol. Sci. 364, 1107-1115. 
Wolf, J. B., Cheverud, J. M., Roseman, C. and Hager, R. (2008). Genome-wide analysis reveals a complex pattern of genomic imprinting in mice. PLoS Genet. 4, e10000091.
Wolff, J. N., Ladoukakis, E. D., Enríquez, J. A. and Dowling, D. K. (2014). Mitonuclear interactions: evolutionary consequences over multiple biological scales. Philos. Trans. R. Soc. B 369, 20130443. 